\title{
Dynamic and distributional properties of prices
}

\author{
Peter D. Kvam (pkvam@indiana.edu) \& Jerome R. Busemeyer (jbusemey@indiana.edu) \\ Department of Psychological \& Brain Sciences, Indiana University \\ 1101 E 10th St, Bloomington, IN 47405, USA
}

\begin{abstract}
Most models of pricing embody a static, deterministic theory of value where the monetary amount people assign to an item is computed as a fixed function of its attributes. Preference reversals - where prices assigned to gambles conflict with preference orders elicited through binary choices - indicate that the response processes going into value assessments are important. In this paper, we additionally show that price responses are sensitive to time pressure, suggesting a dynamic underlying cognitive process. We also show that the elicited price distributions can possess strong positive or negative skew, indicating that diverging information is used to generate buying versus selling and certainty equivalent prices. We develop a computational cognitive model that predicts these continuous distributions of price responses and how they change over time, showing that it can account for the major dynamic and distributional properties of prices and decisions.
\end{abstract}

Keywords: pricing, cognitive model, buying, selling, certainty equivalent

\section{Introduction}

The ability to compute and represent value is a core component of our capacities for making preference-based judgments and decisions. Responses corresponding to monetary value like buying prices, selling prices, or certainty equivalents give insight into these representations by assigning the observed features of an item to a single value on an external scale. In turn, these prices allow us to compare the values assigned to multiple items and establish a preference order over a set of options. They are therefore of central importance to our understanding of preference, serving as a straightforward expressions of item value.

Most common theories of preference based on subjective expected utility (von Neumann \& Morgenstern, 1944; Savage, 1954) or prospect theory (Kahneman \& Tversky, 1979; Tversky \& Kahneman, 1992) posit a static and deterministic view of monetary value, where the attributes of an item are transformed into a utility and multiplied by their (weighted) likelihood to compute an overall value. Decisions are assumed to be made by comparing these computed values and picking the higher value item out of a pair, sometimes with some error in the value estimates or choice rates to capture the associated variability in behavior. However, mounting evidence has suggested that this view of value is insufficient to fully characterize the rich patterns of decision and pricing behavior people exhibit. One example comes from the reversals in apparent preference when monetary gambles are presented as a pair for a decision (where A is chosen over B) versus when they are priced individually (where B is priced higher than A). This phenomenon emphasizes that the method of eliciting value comparisons impacts their apparent preference order (Grether \& Plott, 1979; Birnbaum et al., 2016), suggest- ing that the response processes going into judgments and decisions play a substantial role in manifest preferences. Tversky et al. (1990) explained this gap in terms of the weights assigned to probabilities and payoffs for gambles, suggesting that more attention was paid to probability information when gambles were priced individually.

However, this account does not paint a complete picture of prices and decisions. The price assigned to a gamble also changes as a function of the type of price being elicited, such as the selling price (willingness to accept / WTA) versus buying price (willingness to pay / WTP). Thaler (1980) attributed this divergence to an asymmetric utility between items that are potentially being received (buying) as opposed to given away (selling). Thaler proposed that the different utilities resulted from a shift in the reference point of the buying and selling prices: a failure to buy an item is seen as foregoing a gain, whereas selling an item is seen as a loss from a superior monetary position. Assuming loss aversion in value computation, different weights are assigned to the same payoffs, resulting in diverging prices between buying and selling (an endowment effect).

Such an account potentially explains why there is a mean gap between the two types of prices, but would not account for temporal or distributional differences between them. In this paper, we show why such an account is still insufficient for fully characterizing price judgments. Instead, we develop a theory that expands on previous work by Johnson \& Busemeyer (2005), who proposed that the utilities assigned to dollar values may be consistent across different types of responses but that the response-generating processes lead to diverging decisions and prices. Two key findings in support of this theory - and the one proposed here - are 1) the variance of prices increased as the outcomes of the gambles became less certain (approached 50-50 for 2-outcome gambles); and 2) preference reversals could be explained by a theory of response processes that does not assume different utility mappings for different types of prices.

This paper expands on these results and models by developing a computational cognitive model of pricing that predicts a complete joint distribution of price responses and associated response times. The model proposes that judgments are made by combining initial price biases - based on the type of price elicited, WTA / WTP - with information about each gamble that is considered over time. The prior prices are determined by the perspective of the decision-maker; for example, a seller would naturally be biased to start with a higher prior price than a buyer would. Over time, the decision-maker considers the characteristics of the item they are pricing. In the case of monetary gambles, this involves considering (util- 
ities of) the payoffs and the chance of receiving each one. As they consider different outcomes, their preference state shifts toward the utility of the outcome or attribute currently under consideration. Over time, this yields a dynamic process whereby initial prices give way to prices influenced by the attributes of the items under consideration and support for different price judgments is generated. Once enough support for one of the available judgments is generated, a decisionmaker can halt the information gathering process and make their response.

This characterization of pricing entails a dynamic process in which the distributions of resulting prices depend both on the type of price elicited and the characteristics of the gamble(s) offered. Therefore, we examine how judgments change over time as well as directly examine the distributions of buying, selling, and certainty equivalent ("perspective-neutral") prices. The effect of time is evaluated through by including a time pressure manipulation whereby participants are encouraged to either make their response within a certain time frame (speed emphasis) or take their time in order to make sure they give the most representative price (precision emphasis). Furthermore, we examine the shape of the distributions of price responses in each of the different types of judgments (buying, selling, rating). Finally, we examine how these price judgments compare to other methods of preference elicitation like binary choice, where apparent reversals in valuation have previously been shown (Lichtenstein \& Slovic, 1971).

\section{Methods}

The study was aimed at evaluating the effects of time pressure and price type on the prices people assigned to different gambles. The gambles shown consisted of a payoff (\$0-20) and a chance of winning (0-100\%). A total of 32 Indiana University undergraduate students completed the experiment for course credit. Each participant completed 240 trials of the experiment, spread across 8 conditions: a full factorial design with 4 response conditions and 2 time pressure conditions. The 4 different response conditions were:

1. Buying / WTP, where participants responded to a gamble with the amount of money they would pay to play it;

2. Selling / WTA, where participants gave the amount of money they would accept in order to give up the chance to play the gamble;

3. Certainty equivalent [CE], where participants responded with a price that they believed was equal in value to the gamble (perspective-neutral); and

4. Choice, where participants selected which of a pair of gambles presented on the screen they would prefer to play.

Each trial began when the participant clicked inside a fixation circle presented in the middle of the screen (upper left of Figure ). In the choice trials, two gambles appeared - one on either side of the screen. Participants entered their response (selected the gamble they preferred to play) by clicking the left or the right mouse button to choose the gamble on the left or right side of the screen, respectively. Response times were recorded from the time the gambles appeared to the time the participant clicked one of the mouse buttons.

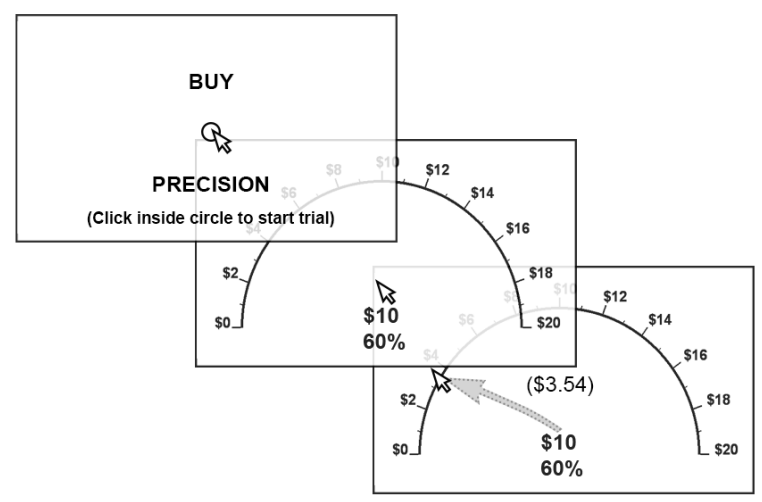

Figure 1: Diagram of the price rating task. Participants were reminded before each trial what type of response they were giving (buy / sell / rate) and whether there was time pressure (speed / precision; left panel). They gave their response by clicking on a semicircular scale (middle / right panels).

On the buying, selling, and rating trials, after clicking the circular fixation, participants instead saw a single gamble appear in the middle of the screen along with a semicircular scale like the one shown in Figure (middle / right panels). They gave their price response using this scale. When their mouse reached the edge of the semicircle, the price indicated by the position of the mouse was shown in parenthesis above the gamble (Figure, right panel). They confirmed this amount and entered their response by clicking on the scale at the desired price. Again, response times were recorded from the time the gamble appeared on-screen until the participant made their response by clicking the mouse. Mouse position during each trial was also recorded but it is not analyzed here.

In addition to the four response type conditions, we also included a time pressure manipulation. This divided trials into two types, speed and precision trials, which were crossed with the response type manipulation for a total of eight conditions. In the speed conditions, participants had to respond in less than 5 seconds for the pricing conditions (buying / selling / $\mathrm{CE}$ ) or less than 2 seconds for the choice condition. They were shown an error message and experienced a short (3.5 second) delay after any trial on which they failed to respond within this time frame. In the precision condition, participants were asked to respond within 10 cents of the desired price in the pricing conditions or prompted to make their selection carefully in the choice condition.

The directions for the time pressure and response type conditions were given at the start of each block of trials, and participants were reminded which condition they were in by text above and below a fixation circle in the middle of the screen 
(Figure, left panel) at the start of every trial as well. Over the course of the study, participants saw 60 different individual gambles (repeated 3 times each) across pricing trials, and 30 pairs of gambles (repeated 2 times each) in the choice trials. Trials were blocked by condition, so that every participant saw 30 trials ( 1 block) of each of the 8 conditions for a total of 240 trials.

\section{Results}

The primary interest was the pricing trials and how participants responded to time pressure. The traditional utility and prospect theory models all predict that each individual can have a different utility and probability weighting function. Therefore, we compare predictions that include time as a factor against a "null" model that includes participant, payoff, probability, and the interaction between payoff and probability as factors predicting price responses. Of course, one could also include response type as a factor in the null model to account for an endowment effect. The null model simply serves as a baseline to see how much, if at all, we can gain by allowing other factors (manipulations) into consideration.

We compared this static / null model against a linear model predicting ratings as a function of response type (Type) and time pressure (Time) as well as the payoff $(\$)$, chance of winning (\%), and their interactions. This was done using Bayesian model comparison, which shows the improvement in predictions that was generated by including Time and Type in the linear model. The results are shown in Table. The Bayes factor $\left(\mathbf{B F}_{\text {Inclusion }}\right)$ reflects the posterior odds of the model including the effect in the left-hand column relative to the null model, and the posterior probability that the factor should be included (P(incl|data) (Rouder et al., 2012). This model used the default priors give in JASP (JASP Team, 2017), which gives equal marginal prior probability to each individual factor and the joint probability of the set of factors (unweighted product) as the prior for all interactions.

The Bayes factors showed strong support for effects of both rating type (Type) and time pressure (Time), as well as an interaction between trial type and the payoff of the gamble (Type*\$). In general, buying prices were lower than selling prices and ratings, as we should expect given theories of endowment or reference point adjustment (Thaler, 1980). While selling and buying prices were higher under time pressure (speed), certainty equivalents appeared relatively stable across time (Figure ).

These results clearly emphasize the importance of time pressure, illustrating that the prices people assign to gambles vary as a function of when they make them. It therefore provides support for using a dynamic model of the judgment process like the one we propose.

Another critical feature of the price responses we observed in the experiment is their distributions, and more specifically how these distributions changed based on the type of trial. The mean prices were clearly higher in the selling (and rating) trials than the buying trials, but this could be due to means

\begin{tabular}{lll} 
Effects & $\mathbf{P}($ incl $\mid \mathbf{d a t a})$ & $\mathbf{B F}_{\text {Inclusion }}$ \\
\hline Time & 0.984 & 4.882 \\
Type & 1 & $3.149 \mathrm{e}+13$ \\
Time*Type & 0.048 & 0.038 \\
Time*\% & 0.195 & 0.142 \\
Time*\$ & 0.126 & 0.084 \\
Type*\% & 0.055 & 0.034 \\
Type*\$ & 0.874 & 4.057 \\
Time*Type*\% & $3.809 \mathrm{e}-5$ & $1.904 \mathrm{e}-4$ \\
Time*Type*\$ & $3.403 \mathrm{e}-4$ & 0.002 \\
Time*\%*\$ & 0.002 & 0.006 \\
Type*\%*\$ & 0.002 & 0.008 \\
Time*Type*\%*\$ & $2.174 \mathrm{e}-10$ & $1.804 \mathrm{e}-8$
\end{tabular}

Table 1: Posterior probability $(P($ incl $\mid$ data $))$ and Bayes factor $\left(B F_{\text {Inclusion }}\right)$ for inclusion of time pressure (Time), trial type (buying / selling / CE; Type), and interaction factors relative to a "null" model that included only payoff (\$), probability $(\%)$, and their interaction.

shifts in a symmetric distribution or changes in the relative skew of buying and selling prices. The latter seems to be the case, indicated by the diverging distributions for buying, selling, and CE prices.

Three illustrative examples from the 60 gambles participants rated are shown in Figure 3. Comparing the location of the median (dots in Figure 3) against the mean (dashed vertical lines in Figure 3) allows us to examine the skew of the distributions. These distributions of prices show a few important properties that appeared consistently across gambles in the experiments. The first is the order of different types of prices - by both metrics of central tendency, buying prices were lowest, $\mathrm{CE}$ prices in the middle, and selling prices the highest.

The second thing to note is the skew of the price responses. Buying prices were almost always right-skewed, with the median (and mode) below the mean buying price. At low chances of winning (bottom panel of Figure 3), selling and CE prices were also right-skewed, but this changed as the chance of winning increased (middle and top panels of Figure 3). While CE prices tended to have a more rightward skew in general, both selling and CE prices showed a strong left skew when chances of winning were high. In these cases, they piled up around the potential payoff amount, with almost no responses above the payoff. As we might expect, there were very few responses above the maximum payoff, and those that were can largely be attributed to motor error.

Finally, the high-variance gambles (with chances of winning closer to $50 \%$ ) like the one shown in the middle panel of Figure 3 showed higher variance in price distributions. The variance in anticipated outcomes translated into variance in represented value, indicating that shifts in the probability attribute produce distributional changes beyond the mean shift in price we would expect from traditional utility models. 


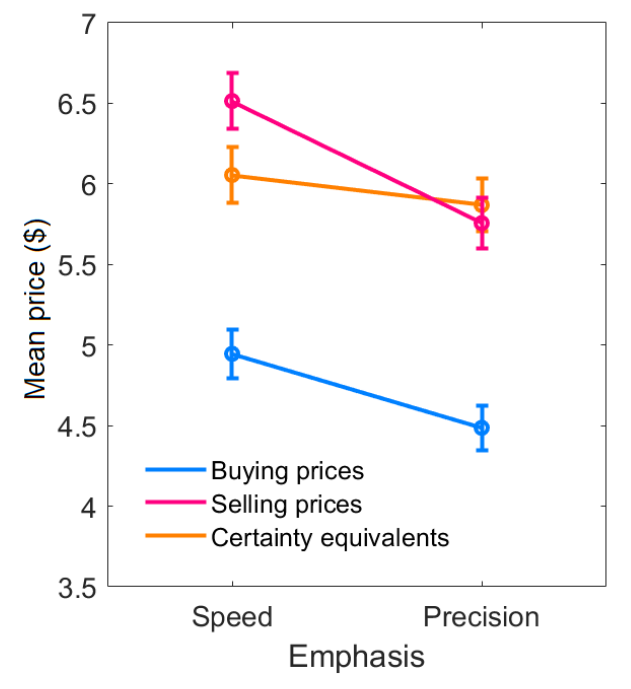

Figure 2: Mean buying (WTP), selling (WTA), and value estimation (rating) judgments as a function of time pressure. Error bars indicate \pm 1 unit of standard error.

\section{Modeling}

Given the multifaceted character of the price data, what sort of theory can account for the impacts of time, type of price response, payoffs, and probabilities of winning? The results of the study suggest that we need a dynamic theory that produces an interaction between the time pressure, type of price, and features of the gambles. Here we propose such a model, where response type produces an initial bias toward different prices, which is in turn integrated with gamble information over time to produces shifts in prices. Over time, this generates support for different prices, and a selection is made when sufficient support for one of the prices has been generated while considering the gamble (threshold).

Formally, the model specifies a starting point $s$ for each pricing process, which depends on the buying, selling, or CE price type manipulation. The starting price is determined by a random draw from a potential distribution of initial prices. In the model, these starting point distributions are set by 3 parameters. The first 2 specify a beta distribution $\operatorname{Beta}\left(\beta_{1}, \beta_{2}\right)$ over initial price values $s_{\beta}$ ranging from the minimum payoff of the gamble (in this case, \$0) to its maximum payoff. The third specifies the strength of this initial belief, as a uniform distribution $\operatorname{Unif}\left(0, s_{v}\right)$. The idea here is that participants will vary in both the prices they are willing to pay / accept before considering the gamble as well as the strength of their convictions about these prices. For example, they could have a strong bias for high prices (starting point $[0, .8]$ ) or a weak bias for low prices (starting point $[.1,0]$ ). Put together, these 3 parameters generate a 2-dimensional starting point distribution for $s$, shown as the shaded region on the left of Figure 4.

Over time, the initial price a person is willing to give can be adjusted as they consider the payoffs and the probabili-
$\$ 9.00,95 \%$

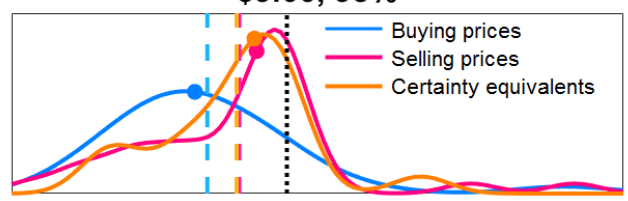

$\$ 15.00,77 \%$

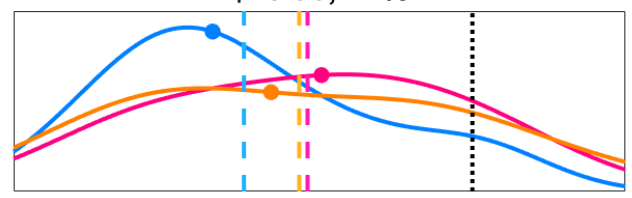

$\$ 20.00,20 \%$

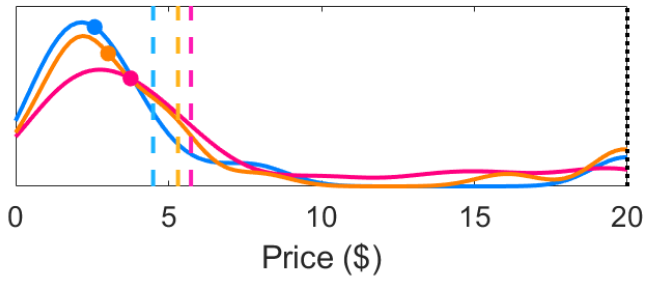

Figure 3: Distributions of pricing judgments for three example gambles, generated from an optimal Gaussian kernel density estimator passed over the observed judgments. Buying prices are shown in blue, selling in pink, and CE prices in orange. The means (dashed vertical lines) and medians (dots on the distributions) of these distributions are shown in corresponding colors to illustrate the skew.

ties of the gamble. The model suggests that a person sequentially updates their valuation by mentally simulating the potential outcomes of the gamble. As they think about receiving an outcome, their representation of the value of the gamble moves toward the utility of that outcome. For example, say a person is considering a gamble with a $50 \%$ chance of winning $\$ 20$. Half the time (for simplicity, we assume no probability weighting in the mental simulation) they think about winning $\$ 20$, and half the time they think about winning $\$ 0$. When they think about winning $\$ 20$, their state moves upward toward high prices. When they think about receiving $\$ 0$, their state moves rightward toward low prices. This model can be thought of as a dynamic version of anchoring and adjustment models (Goldstein \& Einhorn, 1987) - the initial price, impacted by the maximum payoff and the price type, is adjusted according to the potential outcomes of the gamble and their likelihoods as a person mentally simulates the gamble outcomes.

With each new simulated outcome, a person takes a 'step' toward the utility of the simulated payoff. The potential judgments a person can give are arranged in a quarter-circle as in Figure 4 (see Kvam, 2018, for justification for this shape). This arrangement allows simulated outcomes to generate sup- 

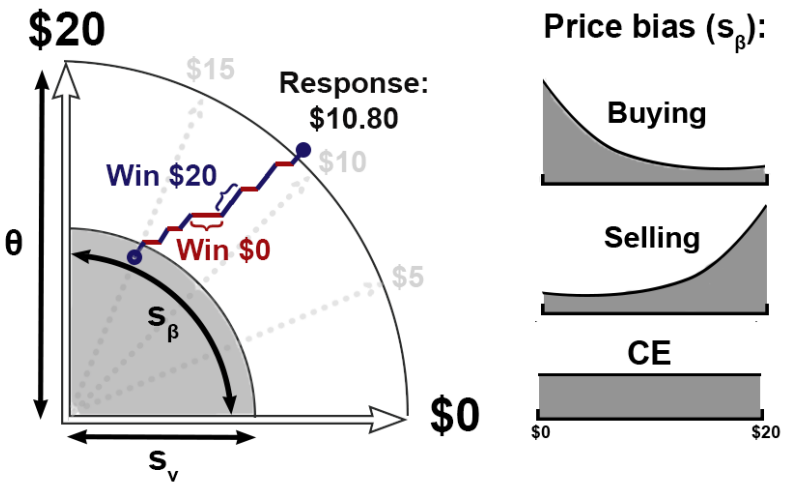

Figure 4: Example trajectory of the price rating process for a decision-maker selling a gamble of $\$ 20,60 \%$. The initial price is set by the prior price distribution given by $s_{v}$ (variation in the strength of the prior) and $s_{\beta}$ (the distribution of prior prices, shown on the right). At each time step, they consider either the possibility of winning \$20 (blue), stepping toward a high price, or failing to win and receiving $\$ 0$ (red), stepping toward a low price $\$ 0$ for the gamble. This proceeds until they hit the threshold $\theta$.

port for multiple prices that are consistent with that payoff - for example thinking about winning \$20 might simultaneously generate support for several high-price responses like $\$ 18.50, \$ 19$, or the other surrounding values. The support for a particular price judgment is given as the component of the represented value along the vector describing that price judgment. If $\$ 20$ is the high value and $\$ 0$ is the low value on the quarter circle, then $\$ 10$ will be at 45 degrees, described by vector $v_{10}=[1,1]$. So the support for a judgment of $\$ 10$ given state $s=[.3, .4]$ would be $\left|\operatorname{proj}_{v_{10}} s\right|=|[.35, .35]|=.495$.

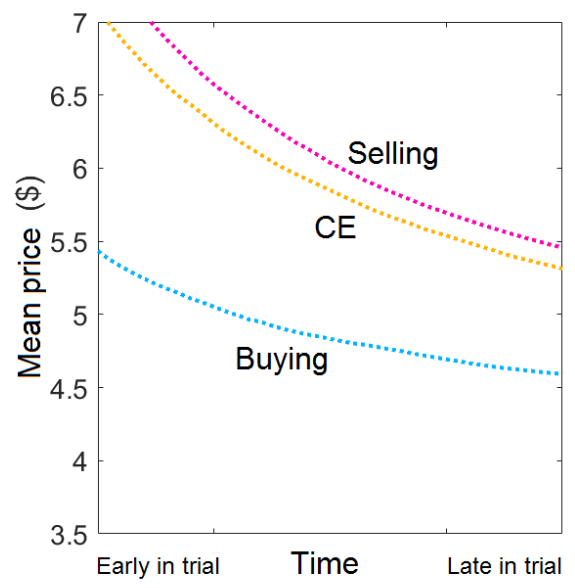

Figure 5: Average trajectory of represented prices as outcomes are mentally simulated over time.

As the decision-maker mentally simulates outcomes, the support for the various prices (component of the price state along the price vectors) shifts over time as shown in Figure 5. On average, the different starting points will wash out over time, bringing the three different price types closer together when participants consider the outcomes longer. Once support for any of the prices exceeds a critical value, the decision-maker responds with the corresponding judgment. This is equivalent to the quarter circular choice boundary with radius $\theta$ shown in Figure 4. The critical value corresponds to the amount of consideration the decision-maker puts into the incoming information before making a decision. As a result, $\theta$ impacts the amount of time it takes a person to give their prices: lower $\theta$ means they will consider the gamble attributes less, giving the initial bias more sway over price responses but resulting in faster responses (further to the left in Figure 5). Higher $\theta$ means that a person will give more consideration to the gamble attributes, reducing the impact of their initial bias in favor of taking more time to look at the features of the gambles (further to the right along the trajectories in Figure 5). Given its parallel role in judgment and binary decision tasks, we should expect the threshold to be higher in a precision-emphasis condition than in a speed-emphasis one, yielding the pattern of results we saw in Figure .

Simulations from this model for the same 3 gambles from Figure 3 are shown in Figure 6. The model captures the most important characteristics of these distributions, including the positive skew of low-probability gambles and the switch to negative skew for high-probability selling price judgments. While model fits are likely to show better fidelity to the observed data, these illustrate in principle that it is capable of capturing the distributional properties of price judgments made in the task.

Of course, there is substantial work to be done in developing and applying the model. we should expect individual differences in all of the parameters for starting points and thresholds, resulting in a mix of generating processes in the group-level data. A complete account of the data should include cognitive model parameters that are fit to each participant separately or hierarchically in addition to varying across conditions, but for now we provide only general pattern of predictions to show that the model can in principle account for the set of results presented here.

\section{Conclusions}

Our findings make a strong case for a dynamic and distributional theory of prices. Time pressure has a strong effect on the price responses people give, as does the type of response (buying, selling, CE). The price responses are also characterized by severely skewed distributions of prices that depend heavily on the type of response elicited. To our knowledge, this is the first time that researchers have explored distributions of price responses, as traditional theory would suggest that they should be tightly distributed (if variable at all) around people's 'true' underlying valuations for the alternatives. Instead, it appears that the underlying price-generating 
$\$ 9.00,95 \%$

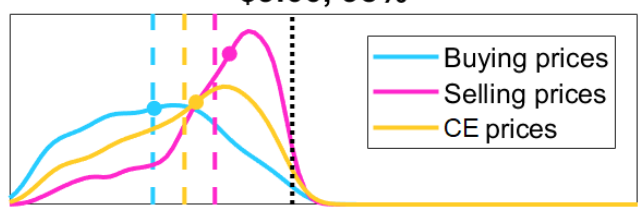

$\$ 15.00,77 \%$

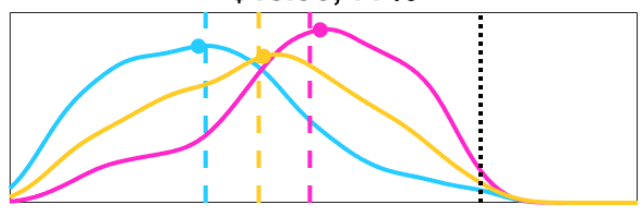

$\$ 20.00,20 \%$

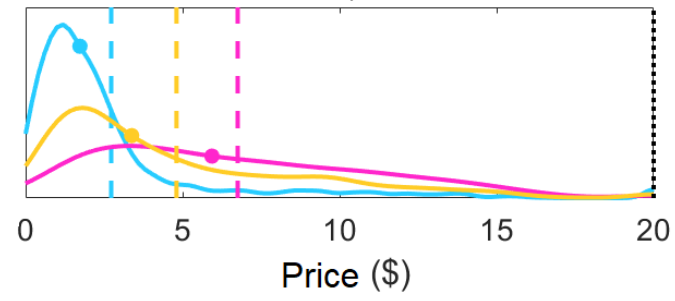

Figure 6: Distributions of pricing judgments generated from the model.

cognitive process produces widely variable and distinct patterns of prices that vary from person to person, buying to selling prices, and speed to precision emphasis.

In order to account for such results, we require a model which is dynamic, stochastic, and produces (near-)continuous distributions of prices. The one we develop here serves each of these requirements, capturing the major characteristics of the price distributions as well as predicting how they change over time. It provides a process-level account of how price judgments are made, and in turn we can compare it to process-level models of how decisions are made. Although we do not have sufficient space here to develop and test the choice model, binary choice consists of a different type of response process where attributes (payoffs and probabilities) are compared between alternatives. It follows closely the binary choice model of decision field theory (Busemeyer \& Townsend, 1993; Johnson \& Busemeyer, 2005). The two models, one for binary choice and the one developed here for pricing, allow for apparent preference reversals where one gamble is priced lower than another when judged individually but chosen over the other when set against one another in binary choice.

Put together, the model provides the first account of price judgment distributions on a continuum, predicts new dynamic phenomena in the production of these judgments, provides an explanation for preferenve reversals, and results in a more complete theory of the cognitive processes that go into price setting.

\section{Acknowledgments}

This work was supported by a grant from the Air Force Office of Scientific Research (FA9550-15-1-0343).

\section{References}

Birnbaum, M. H., Yeary, S., Luce, R. D., \& Zhao, L. (2016). Empirical evaluation of four models of buying and selling prices of gambles. Journal of Mathematical Psychology, 75, 183-193.

Busemeyer, J. R., \& Townsend, J. T. (1993). Decision field theory: a dynamic-cognitive approach to decision making in an uncertain environment. Psychological review, 100(3), 432-459.

Goldstein, W. M., \& Einhorn, H. J. (1987). Expression theory and the preference reversal phenomena. Psychological review, 94(2), 236.

Grether, D. M., \& Plott, C. R. (1979). Economic Theory of Choice and the Preference Reversal Phenomenon. The American Economic Review, 69(4), 623-638. doi: $10.2307 / 1808708$

JASP Team. (2017). JASP (Version 0.8.4)[Computer software]. Retrieved from https:// jasp-stats.org/

Johnson, J. G., \& Busemeyer, J. R. (2005). A Dynamic, Stochastic, Computational Model of Preference Reversal Phenomena. Psychological Review, 112(4), 841-861.

Kahneman, D., \& Tversky, A. (1979). Prospect theory: An analysis of decision under risk. Econometrica, 47(2), 263292.

Kvam, P. D. (2018). A geometric framework for modeling decisions among arbitrarily many alternatives. Submitted for publication.

Lichtenstein, S., \& Slovic, P. (1971). Reversals of preference between bids and choices in gambling decisions. Journal of Experimental Psychology, 89(1), 46-55.

Rouder, J. N., Morey, R. D., Speckman, P. L., \& Province, J. M. (2012). Default Bayes factors for ANOVA designs. Journal of Mathematical Psychology, 56(5), 356-374.

Savage, L. J. (1954). The foundations of statistics. New York, NY: John Wiley \& Sons, Inc.

Thaler, R. (1980). Toward a positive theory of consumer choice. Journal of Economic Behavior \& Organization, 1(1), 39-60. doi: 10.1016/0167-2681(80)90051-7

Tversky, A., \& Kahneman, D. (1992). Advances in prospect theory: Cumulative representation of uncertainty. Journal of Risk and Uncertainty, 5(4), 297-323.

Tversky, A., Slovic, P., \& Kahneman, D. (1990). The causes of preference reversal. The American Economic Review, 204-217.

von Neumann, J., \& Morgenstern, O. (1944). Theory of games and economic behavior. Princeton, NJ: Princeton University Press. 